\title{
Efficient Photoluminescence of Manganese-Doped Two-Dimensional Chiral Alloyed Perovskites
}

\author{
Chao Wang, Linlin Ma, Shiping Wang, Guangjiu Zhao* \\ Tianjin Key Laboratory of Molecular Optoelectronic Sciences, Department of \\ Chemistry, National Demonstration Center for Experimental Chemistry \& Chemical \\ engineering Education, School of Science, Tianjin University, Tianjin 300354, China. \\ E-mail: gjzhao@tju.edu.cn
}




\section{Supporting Information}

\section{EXPERIMENTAL PROCEDURES}

Materials and Sample Preparation

\section{Chemical.}

Lead bromide (PbBr2) 99.0\% Aladdin; Jiuding Chemical (MnBr2) 97.0\%; Hydrogen acid (HBr) $48.0 \mathrm{wt} \%$ in $\mathrm{H} 2 \mathrm{O}$ Aladdin; Hypophosphorous acid (H3PO2) $50.0 \mathrm{wt} \%$ in H2O Aladdin; R-(+)- $\beta$-methylphenethylamine; (R-(+)- $\beta-\mathrm{C} 9 \mathrm{H} 13 \mathrm{~N})$ 98.0\% HEOWNS; Chlorinated phenethylamine salt (PEABr) 99.0\% Xi'an Polymer Light Technology Corp., Ltd. Rural alcohol (C3H8O) GR Aladdin.

\section{Synthesis}

(1) 0-P sample:

It is prepared by solution synthesis, cooling and crystallization method. First weigh $1 \mathrm{mmol} \mathrm{PbBr} 2$ into a $25 \mathrm{ml}$ round-bottom flask, heat and stir $4 \mathrm{ml}$ of $\mathrm{HBr}$ into it in an oil bath until all is dissolved to form a metal halide precursor solution, and then add to the above solution in batches according to the stoichiometric ratio Take the $2 \mathrm{mmol} \mathrm{PEABr}$, and keep the overall solution highly acidic. After all is dissolved, connect a nitrogen protection device, react in an oil bath at $130^{\circ} \mathrm{C}$ for 3 hours, stop stirring, and cool the solution to room temperature, and a large amount of white flakes precipitate The crystals were washed and filtered with isopropanol and placed in a vacuum drying oven at $40^{\circ} \mathrm{C}$ for $12 \mathrm{~h}$, and the samples were dried for later use.

(2) 1-PR sample, 2-PR (Mn0.2) sample, 3-PR (Mn0.5) sample, 4-PR (Mn0.8) sample: The preparation method is similar to the above process. First, weigh (1-x) mmol $\mathrm{PbBr} 2$ and $\mathrm{x}$ mmol $\mathrm{MnBr} 2(\mathrm{x}=0,0.2,0.5,0.8)$ into a $25 \mathrm{ml}$ round-bottom flask, and heat and stir $4 \mathrm{ml}$ of $\mathrm{HBr}$ in an oil bath until all metals are dissolved. Halide precursor solution, and then add $1.6 \mathrm{mmol} \mathrm{PEABr}$ and $0.4 \mathrm{mmol} \mathrm{R}-(+)-\beta-\mathrm{C} 9 \mathrm{H} 13 \mathrm{~N}$ weighed according to the stoichiometric ratio to the above solution in batches, and maintain the high acidity of the overall solution. After all is dissolved, Connect the nitrogen protection device, react in an oil bath at $130^{\circ} \mathrm{C}$ for 3 hours, stop stirring, and cool the 
solution to room temperature. A large number of white flake crystals are precipitated. The crystals obtained by washing and filtering with isopropanol are placed in a vacuum drying oven. Keep it at ${ }^{\circ} \mathrm{C}$ for $12 \mathrm{~h}$, and dry the sample for later use.

\section{Characterization.}

The X-ray diffraction (XRD) patterns of the products were recorded with Bruker Diffraction System using a $\mathrm{Cu} K \alpha$ source $(\lambda=0.15406 \mathrm{~nm})$. The absorption spectrum is tested using our own marine optical platform. Emission spectra were recorded on an Edinburgh Instruments FS5 spectrofluorometer equipped with continuous (150 W) and pulsed xenon lamps.

\section{The PLQE measurement.}

The absolute PLQYs of diluted QD solutions were determined using a fluorescence spectrometer with an integrated sphere ( FS5 ). 


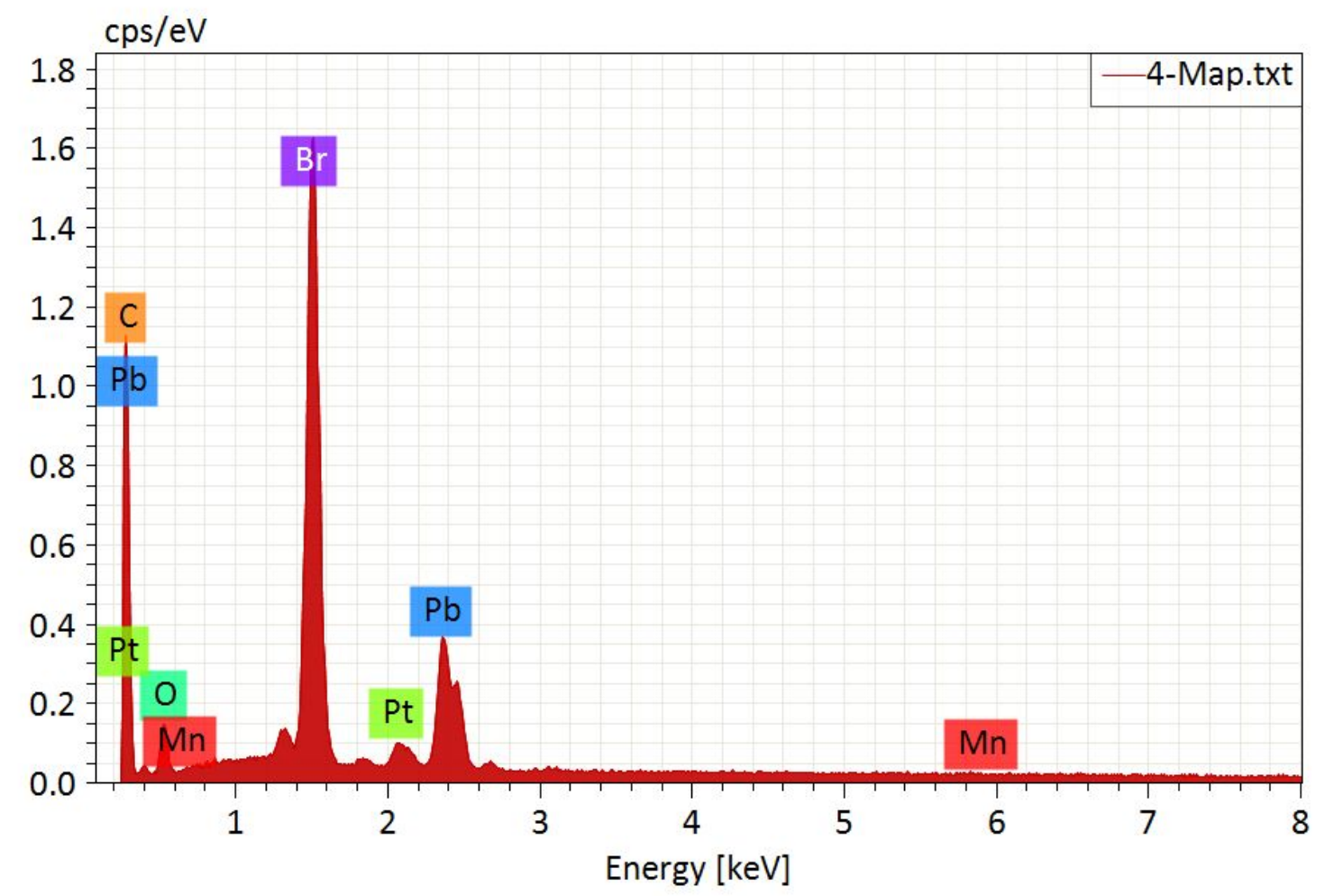

Fig. S1. EDS profile of the system. 

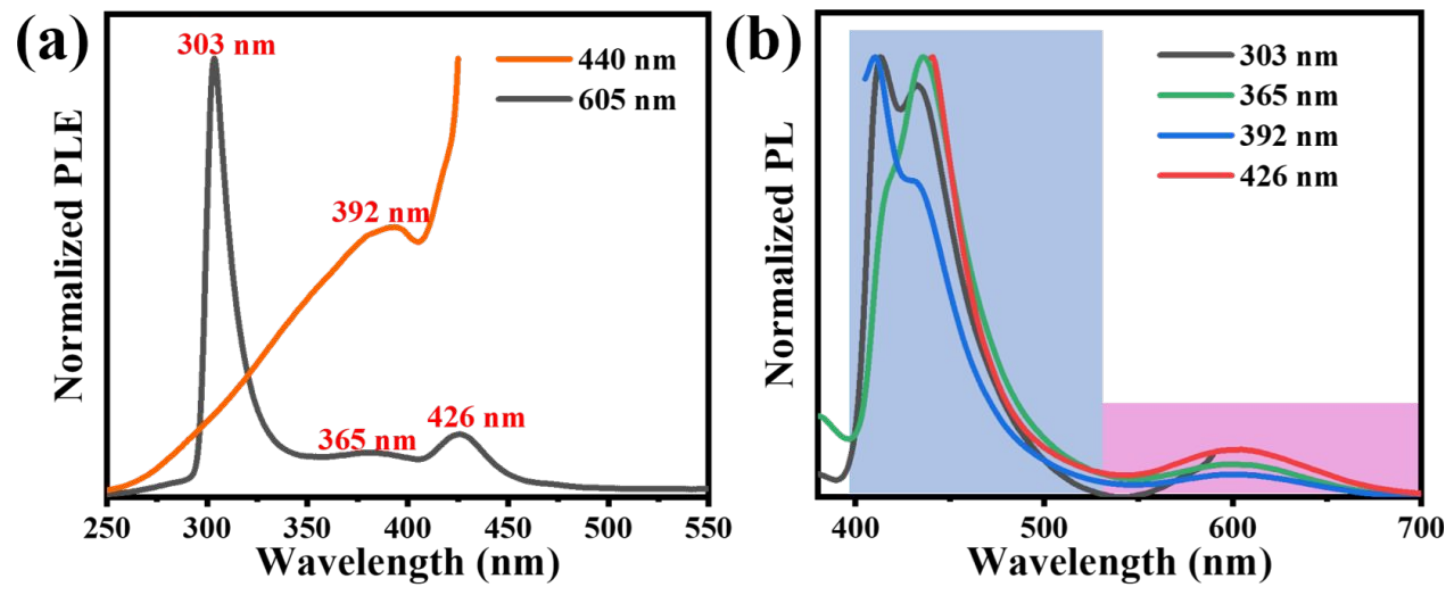

Fig. S2 (a) 3-PR( $\left(\mathrm{Mn}_{0.5}\right)$ excitation spectra measured at two emission peaks; (b) Emission spectra of 3-PR $\left(\mathrm{Mn}_{0.5}\right)$ samples at different excitation wavelengths. 

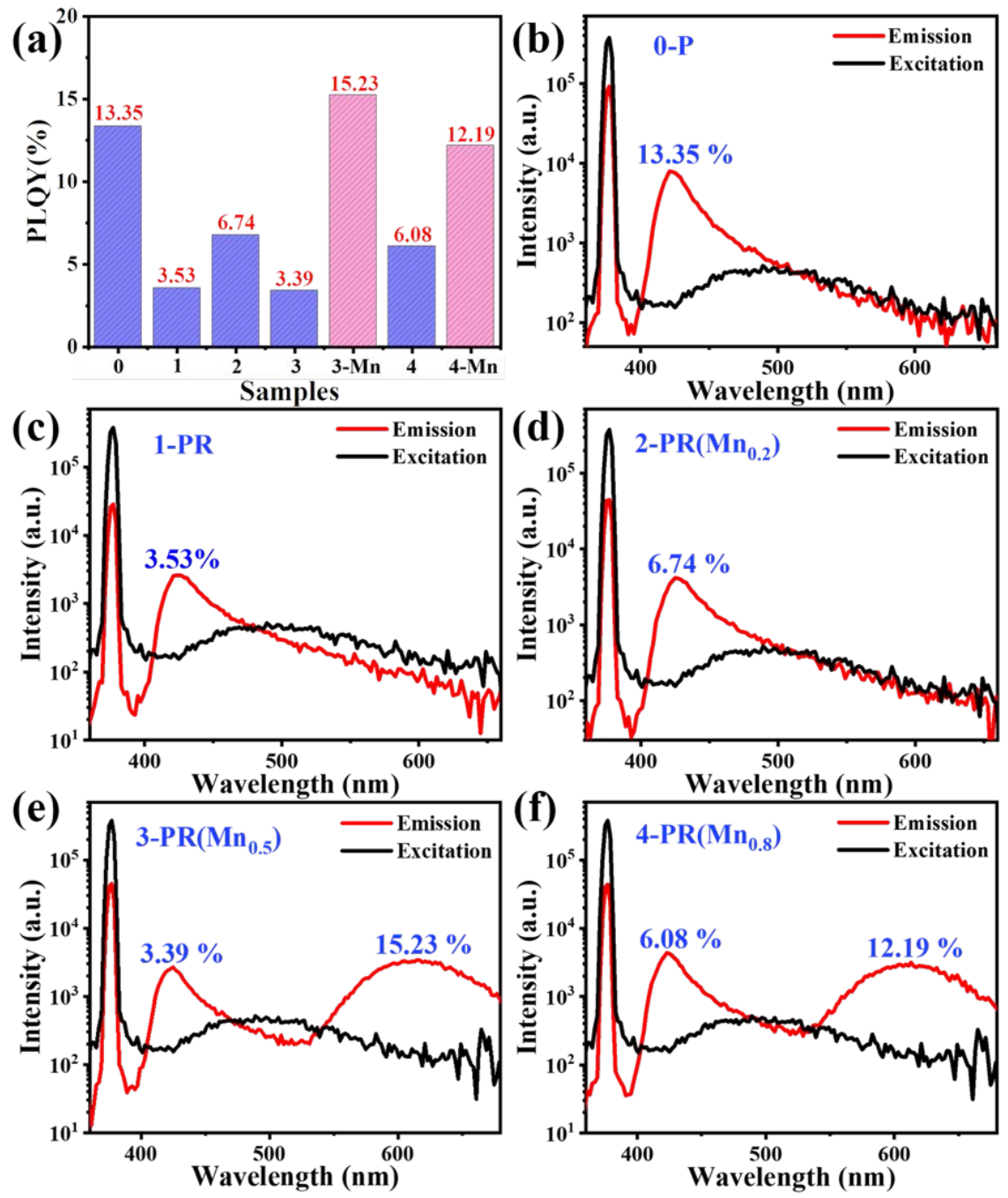

Fig. S3 Characterization of photoluminescence quantum yields of the five samples: (a) Histogram of PLQE distribution of the five samples at different emission positions. Blue represents host emission and red represents characteristic emission of guest Mn. The reference excitation spectra and emission spectra of five kinds of samples were obtained by integrating spheres espectively :(b) 0-P; (c) 1-PR; (d) 2-PR( $\left(\mathrm{Mn}_{0.2}\right)$; (e) 3-PR( $\left(\mathrm{Mn}_{0.5}\right)$; (f) 4-PR( $\left(\mathrm{Mn}_{0.8}\right)$. 


\begin{tabular}{|c|c|c|c|c|c|c|c|}
\hline Sample & $\tau_{1}$ & $\mathbf{A}_{1}$ & $\tau_{2}$ & $\mathbf{A}_{2}$ & $\tau_{3}$ & $\mathbf{A}_{3}$ & $\tau_{\text {ave }}$ \\
\hline 1-PR & $2.02 \mathrm{~ns}$ & $\begin{array}{c}49.8 \\
\%\end{array}$ & $7.99 \mathrm{~ns}$ & $50.2 \%$ & & & $6.78 \mathrm{~ns}$ \\
\hline $2-\operatorname{PR}\left(\mathrm{Mn}_{0.2}\right)$ & $1.69 \mathrm{~ns}$ & $\begin{array}{c}39.5 \\
\%\end{array}$ & $6.61 \mathrm{~ns}$ & $60.5 \%$ & & & $5.89 \mathrm{~ns}$ \\
\hline $\begin{array}{c}3-\operatorname{PR}\left(\mathrm{Mn}_{0.5}\right)-440 \\
\mathrm{~nm}\end{array}$ & $1.41 \mathrm{~ns}$ & $\begin{array}{c}69.1 \\
\%\end{array}$ & $5.41 \mathrm{~ns}$ & $30.9 \%$ & & & $3.89 \mathrm{~ns}$ \\
\hline $\begin{array}{c}3-\operatorname{PR}\left(\mathrm{Mn}_{0.5}\right)-605 \\
\mathrm{~nm}\end{array}$ & $75.4 \mu \mathrm{s}$ & $\begin{array}{c}2.80 \\
\%\end{array}$ & $472 \mu \mathrm{s}$ & $43.7 \%$ & $954 \mu \mathrm{s}$ & $53.5 \%$ & $813.1 \mu \mathrm{s}$ \\
\hline $\begin{array}{c}4-P R\left(M_{0.8}\right)-439 \\
n m\end{array}$ & $1.66 \mathrm{~ns}$ & $\begin{array}{c}63.9 \\
\%\end{array}$ & $4.88 \mathrm{~ns}$ & $36.1 \%$ & & & $3.66 \mathrm{~ns}$ \\
\hline $\begin{array}{c}4-P R\left(M_{0.8}\right)-596 \\
n m\end{array}$ & $197 \mu \mathrm{s}$ & $\begin{array}{c}16.5 \\
\%\end{array}$ & $682 \mu \mathrm{s}$ & $83.5 \%$ & & & $655.8 \mu \mathrm{s}$ \\
\hline
\end{tabular}

Table 1 Time-resolved fluorescence spectrum fitting results of 1-PR, 2-PR $\left(\mathrm{Mn}_{0.2}\right), 3-\mathrm{PR}\left(\mathrm{Mn}_{0.5}\right)$, 3-PR( $\left(\mathrm{Mn}_{0.5}\right), 4-\mathrm{PR}\left(\mathrm{Mn}_{0.8}\right), 4-\mathrm{PR}\left(\mathrm{Mn}_{0.8}\right)$ samples.

The formula is $\tau$ ave $=\frac{A 1 \times \tau 1^{2}+A 2 \times \tau 2^{2}}{A 1 \times \tau 1+A 2 \times \tau 2}$ 